Carvalho, et. al. Percepção da Imagem Corporal e...

\title{
PERCEPÇÃO DA IMAGEM CORPORAL E COMPORTAMENTO ALIMENTAR: ESTUDO COM ACADÊMICOS DE CENTRO UNIVERSITÁRIO
}

\author{
Mateus Barros de Carvalho ${ }^{1}$ \\ Paula Maria da Silva ${ }^{1}$ \\ Luiza Marly Freitas de Carvalho \\ Antonio Marcos Vaz de Lima² \\ Keila Cristiane Batista Bezerra Lopes ${ }^{2}$
}

\begin{abstract}
Resumo
$\mathrm{O}$ conceito de imagem corporal abrange a maneira com que o indivíduo percebe o próprio corpo, levando em consideração suas partes constituintes, assim como os sentimentos relacionados a essas características. $\mathrm{O}$ ingresso na vida universitária provoca alterações no comportamento alimentar, e a exposição ao novo meio social podem trazer como consequência aumento da insatisfação corporal. Objetivo: investigar a satisfação com a imagem corporal, comportamento alimentar de acadêmicos de um centro universitário. Métodos: Para a coleta de dados foi utilizados os seguintes instrumentos, a avaliação nutricional foi realizada através das medidas antropométricas, com as medidas de peso e estatura, para diagnóstico do estado nutricional utilizou-se o Índice de massa corporal. Para avaliar as variáveis do estudo, foram utilizados os questionários Body Checking Questionnaire. Male Body Checking Questionnaire, Body Shape Questionnaire e EAT-26. Resultados: Segundo o IMC o sexo feminino apresentou maior índice de adequação $(70,4 \%)$ em relação ao sexo masculino $(58,9 \%)$. O questionário EAT-26 mostrou que no sexo masculino $85 \%$ dos acadêmicos de engenharia apresentaram risco para TAs , em relação ao sexo feminino psicologia teve $89,8 \%$. Conclusão: Quanto ao comportamento alimentar, as mulheres obtiveram maiores pontuações que os homens, o que sugere uma maior suscetibilidade para o desenvolvimento de TAs.
\end{abstract}

Palavras-chave: Imagem Corporal; Avaliação Nutricional; Comportamento Alimentar

\section{Introdução}

O conceito de imagem corporal abrange a maneira com que o indivíduo percebe o próprio corpo, levando em consideração suas partes constituintes, assim como os sentimentos relacionados a essas características, em síntese, a imagem corporal é um conceito multidimensional que compreende os processos fisiológicos, cognitivos, psicológicos, emocionais e sociais em constante troca mútua.

\footnotetext{
${ }^{1}$ Acadêmicos de Graduação em Nutrição - Centro Universitário Santo Agostinho

${ }^{2}$ Docentes do Curso de Nutrição - Centro Universitário Santo Agostinho
} 
De acordo com Amaral (2015), Imagem Corporal pode ser entendida como um fenômeno multidimensional, caracterizado essencialmente por duas dimensões: a perceptiva relacionada à acurácia do julgamento do tamanho e das formas corporais, e a atitudinal que reflete a influência das crenças, emoções e comportamentos em relação ao corpo.

A satisfação corporal é um constituinte afetivo da imagem corporal que permite o adequado desempenho emocional e social do indivíduo perante ao meio social. Satisfação corporal e auto percepção são fatores primordiais na auto aceitação das pessoas e podem gerar atitudes que interferem no seu convívio social. Por outro lado, insatisfação com o corpo acarreta sentimentos e pensamentos negativos quanto à sua aparência, influenciando o equilíbrio emocional e bem-estar psicológico e consequentemente a qualidade de vida.

O ingresso na vida universitária provoca alterações no comportamento alimentar, e a exposição ao novo meio social podem trazer como consequência aumento da insatisfação corporal. Estudos têm sido realizados no sentido de avaliar a insatisfação com o corpo e também as atitudes alimentares inadequadas em universitários, porém a associação da checagem corporal com essas variáveis ainda é pouco explorada pelos pesquisadores.

A entrada na Universidade é uma etapa marcada por intensas mudanças na vida do estudante, representando, para muitos, o momento em que terá que responsabilizar-se por sua alimentação. Dessa forma, vários fatores podem influenciar o comportamento alimentar, resultando em práticas que podem gerar riscos à saúde (ALMEIDA et al, 2013).

Os universitários normalmente apresentam um padrão de sono irregular, caracterizado por modificações no horário de início e término. Durante a semana o sono possui menor duração em relação aos finais de semana, pois os alunos passam por privações de sono durante os dias de aula ou trabalho. Essas irregularidades repercutem negativamente na saúde desses jovens, comprometendo a atenção, a memória, a capacidade de resolução de problemas e, principalmente, o desempenho acadêmico (ARAÚJO et al, 2013).

Os padrões estéticos vigentes nas sociedades contemporâneas, que ditam que os corpos, para serem belos, devem ser magros ou musculosos e definidos, têm feito com que os indivíduos se preocupem excessivamente com sua aparência e como a mesma é vista pelos outros. Isto, por sua vez, contribui para a existência de insatisfações com a imagem corporal, visto que esses padrões são, muitas vezes, inatingíveis (ALVES, 2009).

Desta forma, o presente estudo teve como objetivo investigar a satisfação com a imagem corporal, comportamento alimentar de acadêmicos de Centro Universitário de Teresina-PI. 


\section{Metodologia}

\section{Caracterização do estudo}

Trata-se de estudo de campo com abordagem transversal quantitativo e descritivo, foi realizado em Centro Universitário localizado na zona urbana e região sul da cidade de TeresinaPiauí.

\section{Aspectos éticos}

A pesquisa foi aprovada pelo Comitê de Ética e Pesquisa - CEP do UNIFSA e tem por número do CAAE 91003018.5.0000.5602 e Parecer de aprovação: 2.799.159 (Anexo 01), todos os acadêmicos que fizeram parte da pesquisa foram esclarecidos sobre os objetivos e metodologia da pesquisa, onde todos assinaram o Termo de Consentimento Livre e Esclarecido (TCLE), de acordo com o regulamento em pesquisa com seres humanos do Conselho Nacional de Saúde/Ministério da Saúde - Resolução No 466, de 12 de dezembro de 2012.

\section{Participantes}

A amostra foi determinada pelo programa Sample Size Calculator by Raosoft. Inc. disponível gratuitamente no endereço eletrônico www.Raosoft.com. O nível de significância de 95\%. O estudo foi realizado com acadêmicos de um Centro Universitário, com população total de 1763 acadêmicos e amostra total de 315 indivíduos distribuídos nos cursos de Engenharia Civil, Psicologia e Educação Física.

A literatura, atualmente, aponta que há uma maior prevalência de transtornos alimentares em acadêmicos de cursos nos quais a aparência física é de grande importância, como o de educação física, enfermagem, nutrição. Por esse motivo a inovação em correlacionar com cursos de outras áreas.7

\section{Procedimentos Metodológicos}

\section{Avaliação do estado nutricional}

Foram utilizados alguns dados antropométricos para a realização do diagnóstico nutricional dos participantes, o procedimento ocorreu de acordo com as orientações da Vigilância Alimentar e Nutricional - SISVAN, seguindo os parâmetros base do IMC, de acordo com a Organização Mundial da Saúde - OMS. 
Foi necessário pesar, com o auxílio de uma balança digital e medir a estatura, com a fita métrica, de cada participante e em seguida feito o cálculo do Índice de Massa Corporal - IMC, seguindo os pontos de cortes determinados para o SISVAN.

O cálculo do IMC que foi utilizado: Índice de Massa Corporal (IMC) $=$ Peso (kg)/Altura ${ }^{2}$ (m).

Quadro 01: Pontos de corte estabelecido para adultos

\begin{tabular}{|c|c|}
\hline IMC $\left(\mathbf{k g} / \mathbf{m}^{2}\right)$ & DIAGNÓSTICO NUTRICIONAL \\
\hline$<18,5$ & Baixo Peso \\
\hline$\geq 18,5 \mathrm{e}<25$ & Adequado ou Eutrófico \\
\hline$\geq 25 \mathrm{e}<30$ & Sobrepeso \\
\hline$\geq 30$ & Obesidade \\
\hline
\end{tabular}

Fonte: (WHO, 1995)

\section{Checagem corporal}

Para avaliar os comportamentos de checagem do corpo no sexo feminino, foi utilizado o Body Checking Questionnaire (BCQ), questionário composto por 12 questões de autorrelato em escala na forma Likert de cinco pontos $($ Nunca $=1$ a Muito frequentemente $=5)$. $\mathrm{O}$ escore varia de 12 a 60 pontos. Quanto maior o escore, mais frequente é o comportamento de checagem corporal.

A avaliação da checagem corporal dos indivíduos do sexo masculino foi realizada pelo Male Body Checking Questionnaire (MBCQ). O MBCQ é um instrumento de autorrelato com 19 itens, respondidos em escala na forma Likert de cinco pontos (Nunca $=1$ a Muito frequentemente $=5$ ), em que os indivíduos, no atual momento, avaliaram a frequência em que adotam determinados comportamentos. O escore varia de 19 a 95 pontos. Quanto maior a pontuação, mais frequentes são os comportamentos relacionados à checagem corporal.

\section{Comportamento alimentar}

Foi avaliado pela aplicação da versão traduzida do (EAT-26). Onde esse questionário é composto por 26 questões de autorrelato, onde respondidas em uma escala Likert de pontos que varia de sempre ( 3 pontos) a nunca ( 0 ponto), exceto a questão 25 , que apresenta pontuação inversa. Escore superior ou igual a 21 pontos salienta possíveis comportamentos de risco para o transtorno alimentar. 


\section{Satisfação com a imagem corporal}

Para avaliar a satisfação e as preocupações com a forma do corpo, foi utilizado o Body Shape Questionnaire (BSQ) em sua versão validada para uma população de universitários brasileiros de ambos os sexos. O questionário é composto por 34 questões, em escala na forma Likert de seis pontos $($ Nunca $=1$ a Sempre $=6$ ), em que o avaliado foi instruído com que frequência vivenciou os eventos propostos pelas alternativas.

\section{Análise de Dados}

Os dados coletados foram armazenados em planilhas do Excel e posteriormente exportados para o banco de dados criado no software Statistical package for The social Sciences (SPSS) versão 16.

Foi realizado o teste de normalidade Kolmogorov- Smirnov no sofware R, para as pontuações obtidas por cada entrevistado segundo os questionários checagem corporal e comportamento alimentar. Os resultados obtidos foram dados de acordo com a pontuação dos escores e seguem a distribuição normal (com exceção do escore do comportamento alimentar que apresenta pontuação inversa), com isso, a associação entre o curso e a pontuação dos questionários foi através do teste t Student para amostras independentes, ao nível de 5\% de significância, já para o comportamento alimentar dos estudantes de educação física, usou-se o teste não paramétrico conhecido como U- de Mann Whitney para amostras independentes, ao nível de 5\% de significância.

\section{Resultados e Discussão}

Os resultados foram agrupados por sexo, comparando homens e mulheres de acordo com algumas variáveis. Dos 315 estudantes avaliados 146 foram do sexo masculino e 169 do sexo feminino. 


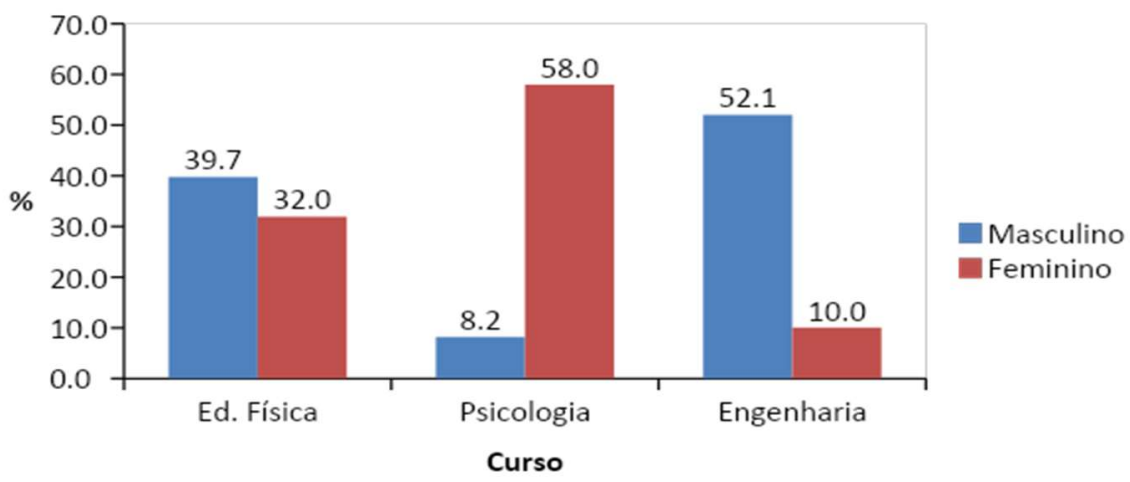

Figura 1 - Distribuição da amostra por curso e sexo, Teresina, PI, 2019. Fonte: dados da pesquisa, 2019.

Pode-se observar que do total da amostra, o curso com maior número de participantes foi o curso de Psicologia, com 58\% de participantes do sexo feminino, já o em relação ao sexo masculino, destacou-se o curso de Engenharia Civil correspondente a 52\%. (Figura 1).

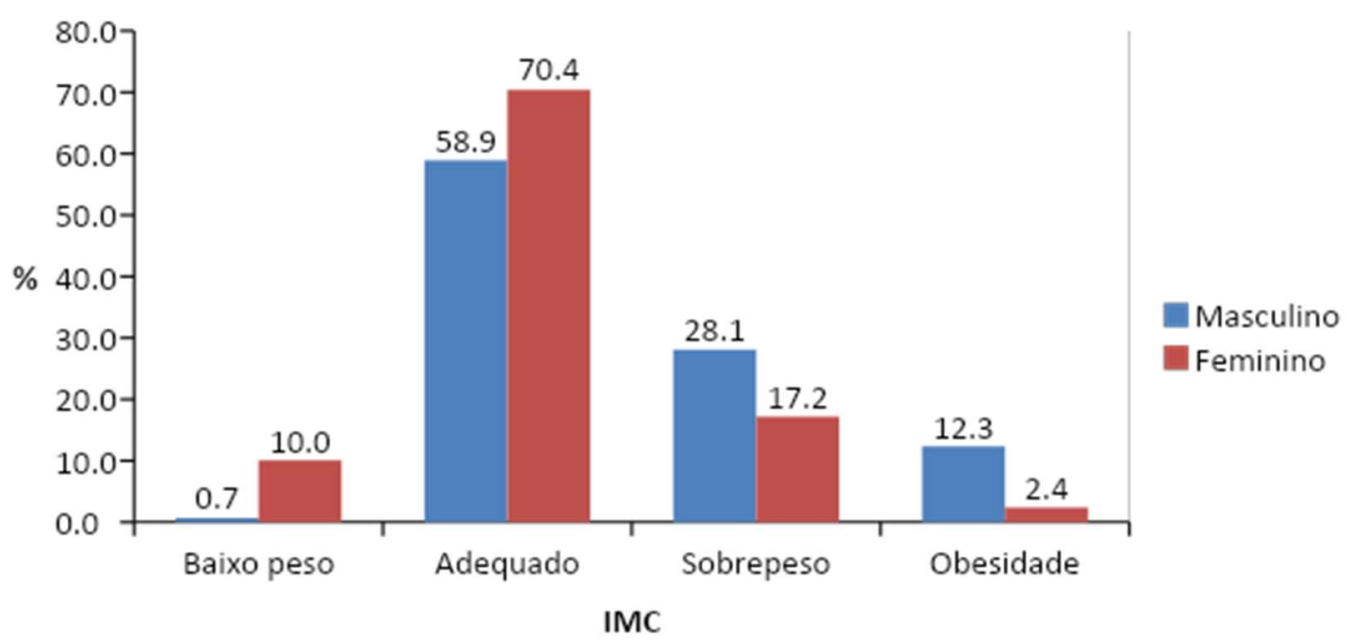

Figura 2 - Classificação do IMC dos participantes, por sexo Teresina, PI, 2019 Fonte: Dados da pesquisa, 2019.

Nota-se que em relação ao IMC, o sexo masculino $(58,9 \%)$ está na classificação adequada do IMC $(>18,5$ e $<25)$, entretanto cerca de 40\% da amostra apresentaram excesso de peso $28,1 \%(n=41)$ e $12,3 \%(n=18)$ obesidade em relação ao IMC, no sexo feminino $70 \%$ $(\mathrm{n}=119)$ apresentaram na classificação adequada do $\operatorname{IMC}(>18,5 \mathrm{e}<25)$, entretanto cerca de $10 \%(\mathrm{n}=17)$ apresentaram baixo peso e $20 \%$ da amostra apresentou excesso de peso $17,2 \%$ $(n=29)$ e $2,4 \%(n=4)$ obesidade (Figura 2$)$. 
Resultados semelhantes foram encontrados Gasparetto, et al., (2012) observou-se que $64,2 \%$ dos universitários apresentavam-se eutróficos; 2,7\% magreza grau I; 27,7\% com sobrepeso; 3,6\% obesidade grau 1 e 1,7\% obesidade grau 2. A média de IMC foi de 24,04 $\mathrm{Kg} / \mathrm{m}^{2}$. Quanto ao sexo, as mulheres apresentaram média de $\mathrm{IMC}$ de $23,09 \pm 3,46 \mathrm{Kg} / \mathrm{m}^{2}$, enquanto os homens, $26,13 \pm 3,68 \mathrm{Kg} / \mathrm{m}^{2}(\mathrm{p}<0,001)$.

Estudo de Ferreira (2013) avaliou 587 universitários (311 homens) com idade média de 20,98 anos $(\mathrm{DP}=3,65)$. Desses, 77,51\% $(\mathrm{n}=455)$ eram eutróficos, 18,91\% $(\mathrm{n}=111)$ estavam com sobrepeso e $3,58 \%(n=21)$, obesos.

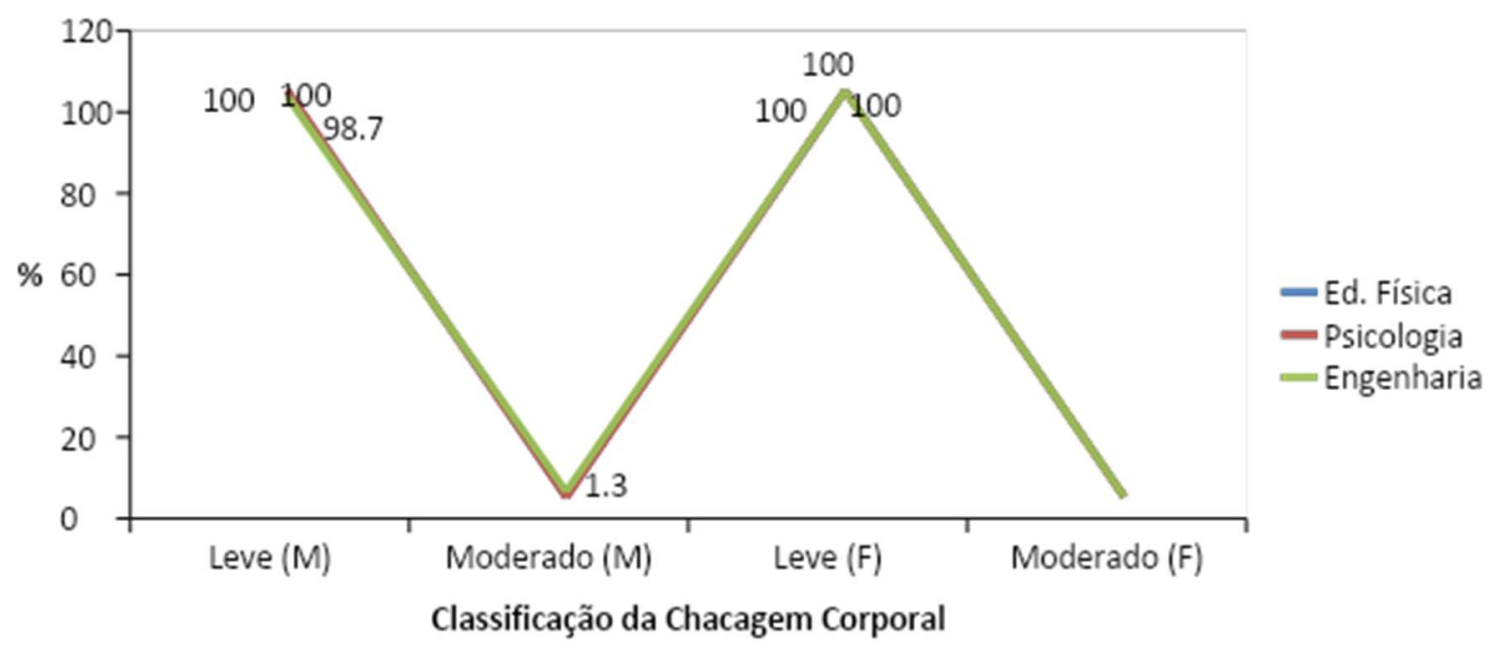

Figura 3 - Classificação da percepção corporal, segundo os cursos dos estudantes, dividido por sexo, Teresina, PI, 2019.

Fonte: Dados da pesquisa, 2019.

Escore $(\mathrm{MBCQ})<90=$ leve, $90-110=$ moderado, $>110=$ intenso, Leve $(\mathrm{H})=$ Class. Leve para os homens, Moderado $(\mathrm{H})=$ Class. Moderado para os homens, Leve $(\mathrm{M})=$ Class. Leve para as mulheres, Moderado $(\mathrm{M})=$ Class. moderado para as mulheres.

De acordo com o estudo de Assis (2013), 9,8\% das mulheres consideravam-se magras e pouco abaixo do peso ideal e 47,5\% consideravam-se acima do peso ideal, sendo que $42,7 \%$ achavam-se com o peso ideal e 57,3\% estavam insatisfeitas com seu peso corporal e, possivelmente, com sua imagem corporal. Sobre a relação entre IMC e peso corporal, os resultados obtidos foram: $24,5 \%$ dos participantes avaliaram seu peso corporal menor do que sua classificação do IMC; $23,2 \%$ dos sujeitos avaliaram seu peso corporal maior do que a 
classificação do IMC; e 52,3\%, ou seja, a maioria, avaliaram seu peso corporal igual à sua classificação do IMC.

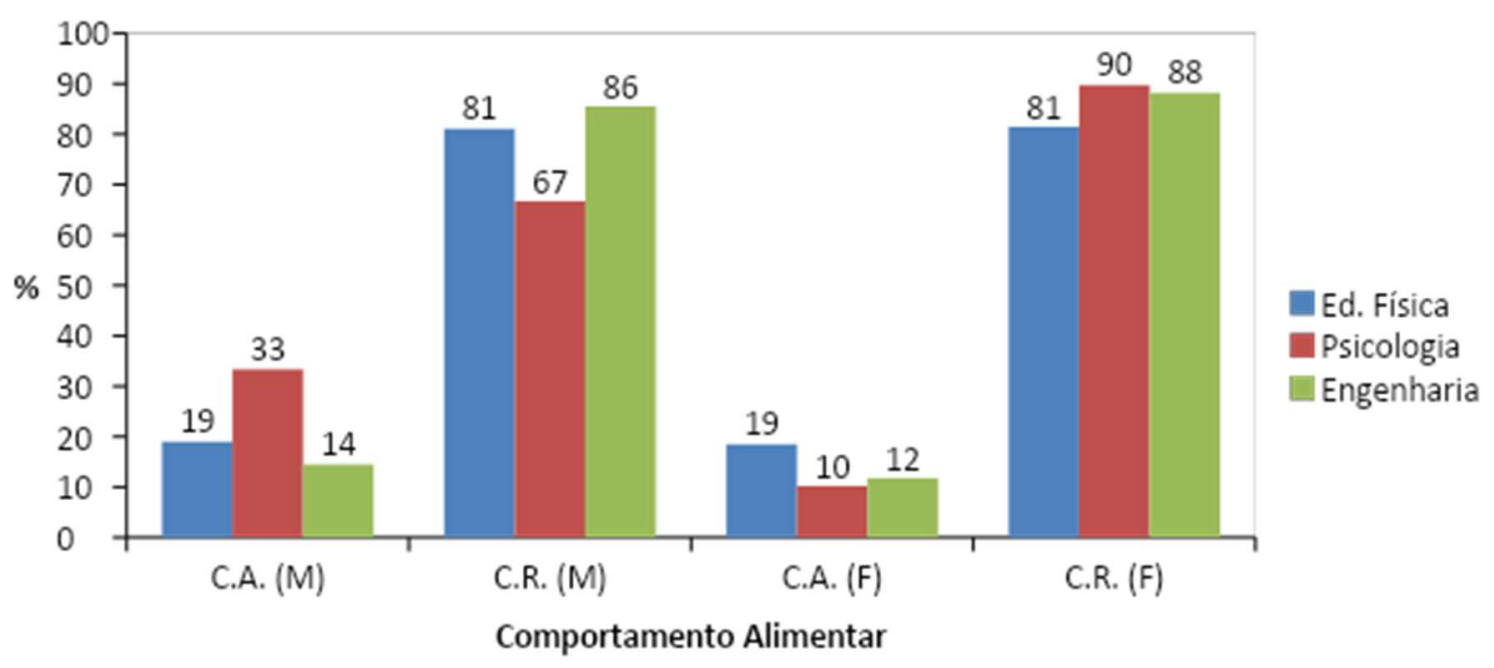

Figura 4 - Classificação do comportamento alimentar, segundo os cursos dos acadêmicos, subdividido por sexo, Teresina, PI, 2019.

Fonte: Dados da pesquisa, 2019.

Escore $($ EAT-26) $<21=$ comportamento aceitável, $>20=$ comportamento de risco, C.A. $(\mathrm{M})=$ Comportamento aceitável para os homens, C.R. $(\mathrm{M})=$ Comportamento de risco para os homens, C.A. $(\mathrm{F})=$ Comportamento aceitável para as mulheres, C.R. $(\mathrm{F})=$ Comportamento de risco para as mulheres.

De acordo com os dados obtidos em relação a comportamento alimentar em indivíduos do sexo masculino pode-se observar que no curso de educação física $81 \%$ apresentaram comportamento de risco, psicologia $66,7 \%$ e engenharia 85,5\%. Já em relação a comportamento alimentar em indivíduos do sexo feminino observou-se que no curso de educação física $81,5 \%$ apresentaram comportamento de risco, psicologia $89,8 \%$ e engenharia $88,2 \%$.

De acordo com o estudo de Laus (2009), os resultados relativos à aplicação do EAT-26 nas estudantes de Educação Física, Publicidade e Administração corroboram dados da literatura. Entretanto, observa-se que, no presente estudo, as estudantes do sexo feminino mostraram um índice de positividade de 54\%.

Estudo de Alvarenga et al., (2011) foi encontrado uma frequência que variou de 23,7\% a 30,1\% em jovens universitárias das cinco regiões do Brasil, utilizando o mesmo instrumento avaliativo (EAT-26), com ponto de corte igual ou superior a 21 pontos. 

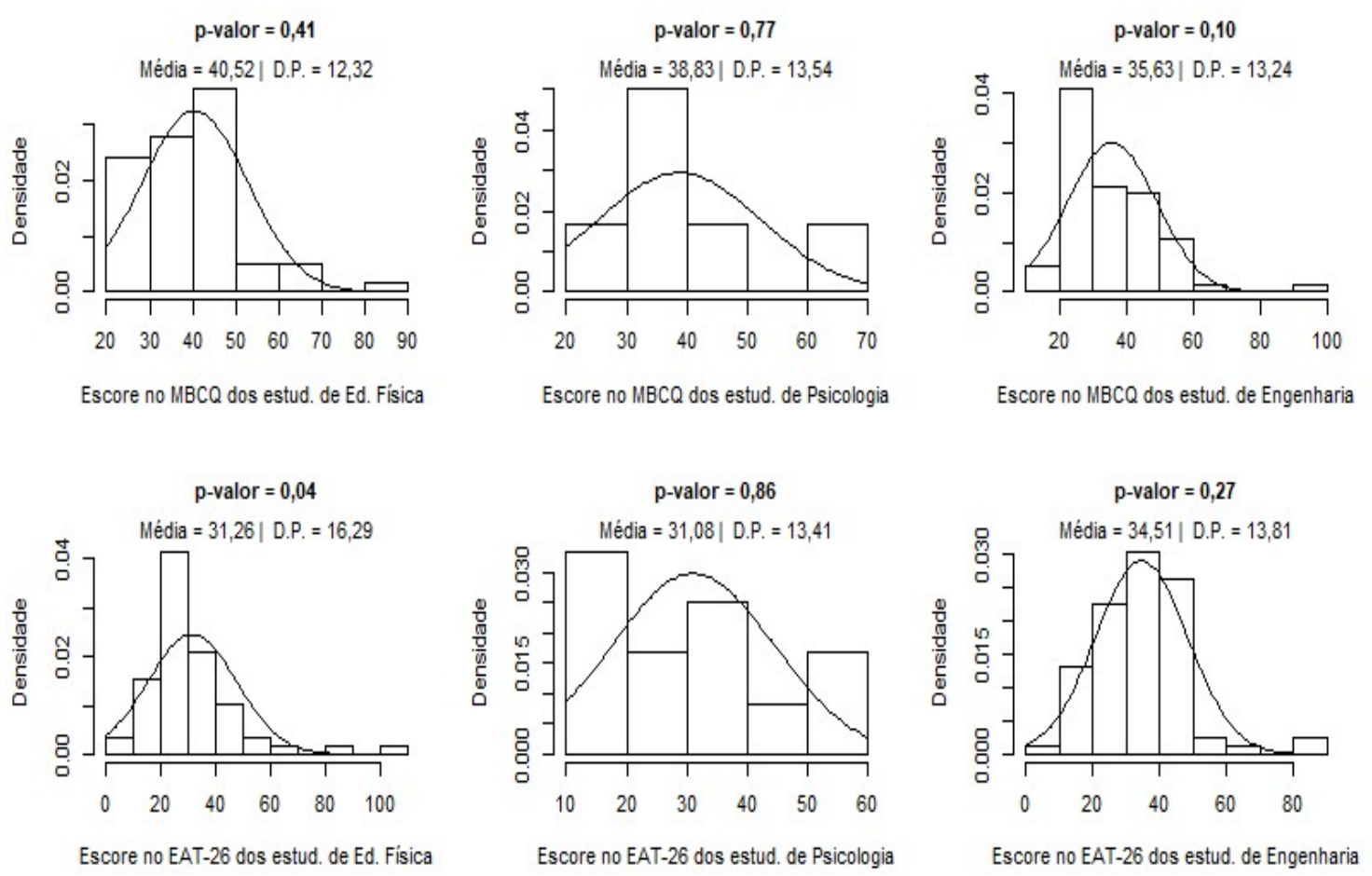

Figura 5 - Verificação dos escores obtidos na checagem corporal e comportamento alimentar dos estudantes por curso. Teresina-PI.

Fonte: Dados da pesquisa, 2019

p-valor $>0,05=$ indicativo que os dados seguem distribuição normal.

Conforme a Figura 5 foi realizado o teste de normalidade Kolmogorov- Smirnov no software R, para as pontuações obtidas por cada entrevistado segundo os questionários checagem corporal e comportamento alimentar.

O resultado obtido é que os escores seguem a distribuição normal (com exceção do escore do comportamento alimentar dos estudantes de educação física), com isso a associação entre o curso e a pontuação dos questionários foi através do teste t Student para amostras independentes, ao nível de 5\% de significância, já para comportamento alimentar dos estudantes de educação física, usou-se o teste não paramétrico conhecido como U- de Mann Whitney para amostras independentes, ao nível de 5\% de significância. 
Tabela 1 - Relação dos valores do Teste t Student entre os cursos e o total de sua pontuação obtida nos questionários MBCQ e EAT-26, Teresina, PI, 2019.

\begin{tabular}{llllll}
\hline Questionários & Curso & N & Média & D.P.* & p-valor** \\
\hline \multirow{2}{*}{ MBCQ } & Ed. Física & 58 & 40,52 & 12,31 & \multirow{2}{*}{0,673} \\
& Psicologia & 12 & 38,83 & 13,54 & \\
\multirow{2}{*}{ EAT-26 } & Ed. Física & 58 & 31,26 & 16,29 & \multirow{2}{*}{0,972} \\
& Psicologia & 12 & 31,08 & 13,41 & \\
\hline \multirow{2}{*}{ MBCQ } & Ed. Física & 58 & 40,52 & 12,31 & \multirow{2}{*}{$\mathbf{0 , 0 3 1}$} \\
& Engenharia & 76 & 35,63 & 13,24 & \\
\hline \multirow{2}{*}{ MBCQ } & Ed. Física & 58 & 31,26 & 16,30 & \multirow{2}{*}{0,214} \\
& Engenharia & 76 & 34,51 & 13,81 & \\
\hline \multirow{2}{*}{ EAT-26 } & Psicologia & 12 & 38,83 & 13,54 & \multirow{2}{*}{0,44} \\
& Engenharia & 76 & 35,63 & 13,24 & \\
\hline
\end{tabular}

Fonte: Dados da pesquisa, 2019

* Desvio padrão; ** p-valor $\leq 0,05$ é indicativo de diferença entre os grupos; a $=$ Utilizou-se o teste não paramétrico U- de Mann Whitney.

De acordo com a Tabela 1, houve diferença significativa entre os cursos de Ed. física e engenharia referente ao questionário MBCQ que é uma avaliação da checagem corporal, ou seja, a um nível de 5\% de significância os estudantes de educação física fazem mais checagem corporal do que os de engenharia. Visto que a literatura, aponta que há uma maior prevalência de transtornos alimentares em acadêmicos de cursos nos quais a aparência física é de grande importância.

Nesse estudo foi possível observar que a relação entre checagem corporal e IMC dos entrevistados do sexo masculino cerca de 98,8\% apresentam risco leve e apenas 1,2\% apresentam risco moderado. Enquanto no sexo feminino $100 \%$ apresentaram risco leve quanto ao nível em que realizam checagem corporal. 
Tabela 2 - Classificação do comportamento alimentar, segundo o resultado do IMC dos estudantes, subdividido pelo sexo, Teresina, PI, 2019.

\begin{tabular}{lllll}
\hline SexolIMC & Baixo peso (\%) & Adequado (\%) & Sobrepeso (\%) & Obesidade (\%)
\end{tabular}

Homens

$\begin{array}{lllcc}\text { C.R. } & 100,0 & 19,8 & 19,5 & 5,6 \\ \text { C.A. } & 0,0 & 80,2 & 80,5 & 94,4\end{array}$

Mulheres

$\begin{array}{lllll}\text { C.R. } & 76,5 & 89,9 & 79,3 & 100,0 \\ \text { C.A. } & 23,5 & 10,1 & 20,7 & 0,0\end{array}$

Fonte: Dados da pesquisa, 2019. Escore $($ EAT-26) $<21=$ comportamento aceitável, $>20=\quad$ comportamento de risco, C.A. = Comportamento aceitável, C.R $=$ Comportamento de risco.

De acordo com os dados obtidos nota-se que a relação entre IMC e comportamento alimentar dos participantes do sexo feminino, baixo peso apresentaram 76,5\% comportamento de risco, já com peso adequado $89,9 \%$ apresentaram comportamento de risco e com sobrepeso apresentaram 79,3\% de comportamento de risco, (Tabela 2).

De acordo com a Figura 7, todos os escores dos homens subdivididos pela classificação do IMC tem distribuição normal. 


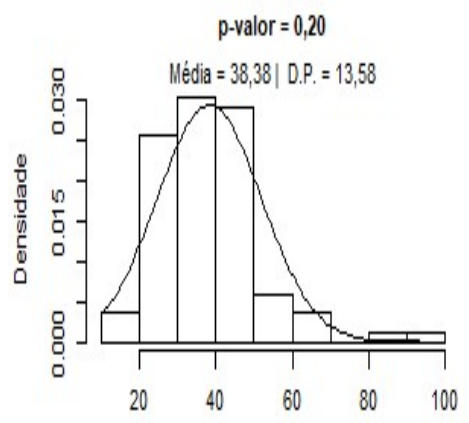

Escore no MBCQ dos estud. com WIC adequado

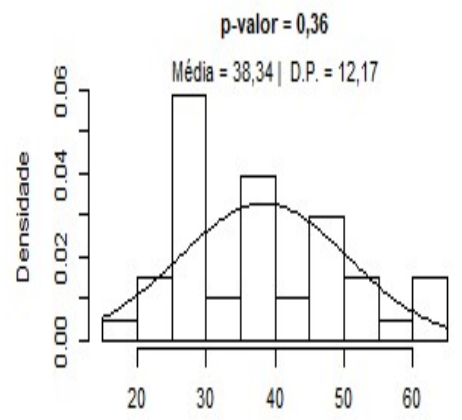

Escore no MBCCQ dos estud. com liC acima do peso

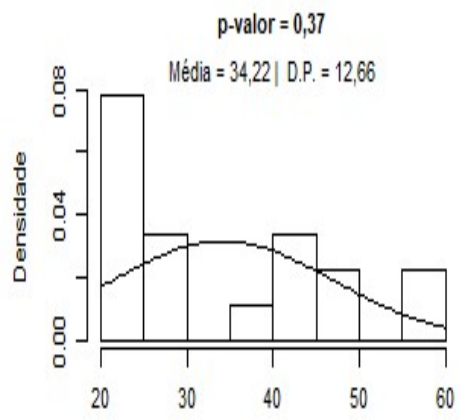

Escore no MBCQ dos estud. com MIC class. como obeso
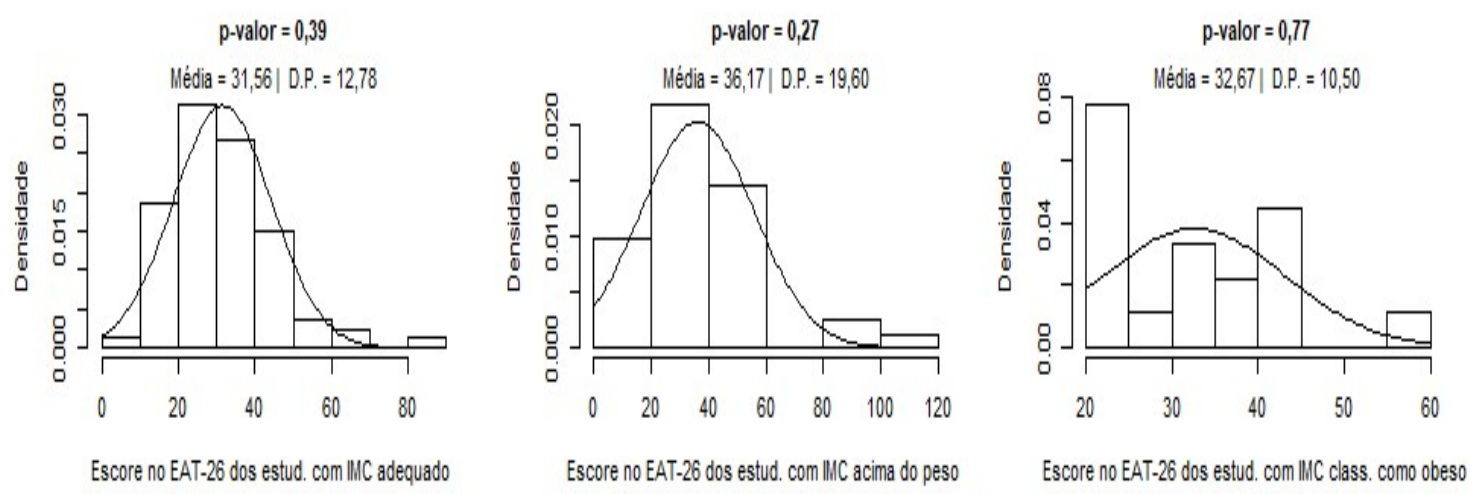

Figura 6- Teste de Kolmogorov- Smirnov para averiguar se os escores obtidos pelos acadêmicos dos cursos de Ed. física, Psicologia e Engenharia, segundo a classificação do IMC, seguem distribuição Normal para a checagem corporal e comportamento alimentar.

Fonte: Dados da pesquisa, 2019. p-valor $>0,05=$ indicativo que os dados seguem distribuição normal.

Tabela 3 - Teste t Student entre o IMC do entrevistado e o total de sua pontuação obtida nos questionários MBCQ e EAT-26, Teresina, PI, 2019.

\begin{tabular}{lllccc}
\hline Questionários & IMC & N & Média & D.P.* & p-valor** \\
\hline \multirow{2}{*}{ MBCQ } & Adequado & 86 & 38,38 & 13,58 & \\
& Sobrepeso & 41 & 38,34 & 12,17 & 0,987 \\
& Adequado & 86 & 31,56 & 12,78 & \\
EAT-26 & Sobrepeso & 41 & 36,17 & 19,60 & 0,115 \\
& & & & & \\
\hline MBCQ & Adequado & 86 & 38,38 & 13,58 & 0,235
\end{tabular}




\begin{tabular}{lccccc} 
& Obesidade & 18 & 34,22 & 12,66 & \\
& & & & & \\
EAT-26 & Adequado & 86 & 31,56 & 12,78 & 0,732 \\
& Obesidade & 18 & 32,67 & 10,50 & \\
& & & & & \\
MBCQ & Sobrepeso & 41 & 38,34 & 12,17 & 0,242 \\
& Obesidade & 18 & 34,22 & 12,66 & \\
\hline \multirow{2}{*}{ EAT-26 } & Sobrepeso & 41 & 36,17 & 13,97 & \\
& Obesidade & 18 & 32,67 & 13,63 & \\
& & & & & \\
\hline
\end{tabular}

Fonte: dados da pesquisa; * Desvio padrão; ** p-valor $\leq 0,05$ é indicativo de diferença entre os grupos.

Na Tabela 3, foi relacionado à classificação do IMC com a total da pontuação dos questionários, não utilizamos a classificação baixo peso, pois a sua frequência na amostra foi baixa $(\mathrm{n}=1)$, o que impossibilita a realização do teste t Student.

Observe que não tivemos nenhum $p$ - valor significativo $(p \leq 0,05)$, então com um nível de 5\% não temos relação entre a classificação do IMC e a pontuação nos questionários.

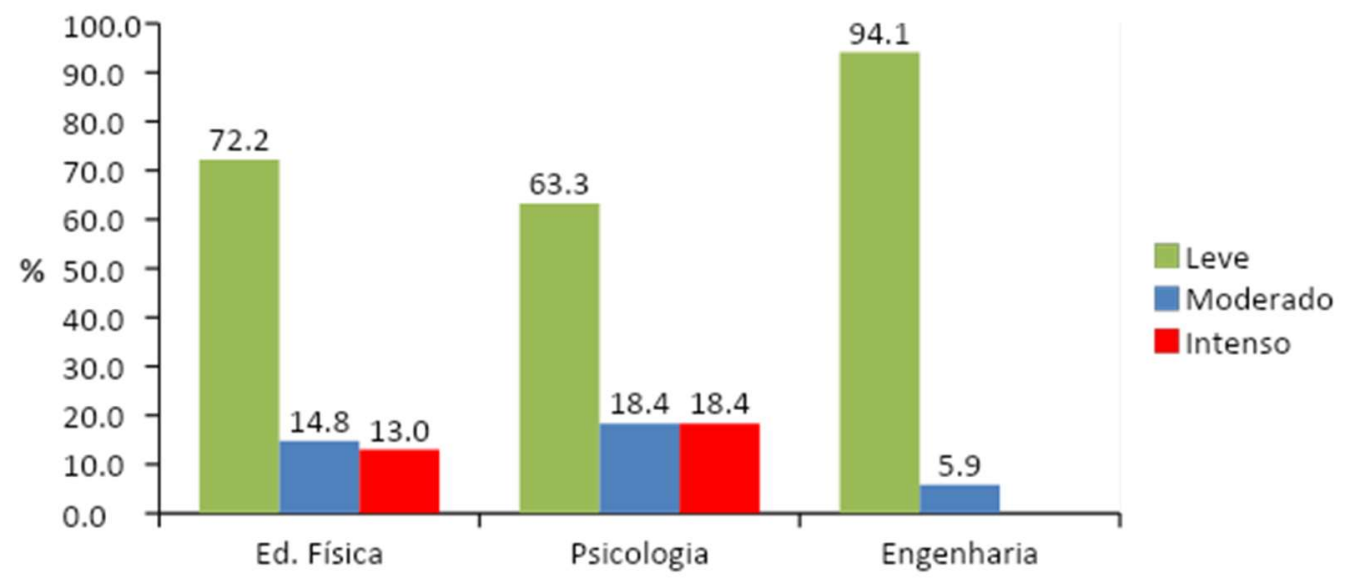

Figura 7 - Classificação da satisfação corporal, segundo os cursos, do sexo feminino, Teresina, PI, 2019.

Fonte: dados da pesquisa,2019. Escore (BCQ) $<90=$ leve, $90-110=$ moderado, $>110=$ intenso. 
De acordo com os dados obtidos através da aplicação do questionário de satisfação corporal pode-se observar que no curso de educação física $72,2 \%$ apresentaram satisfação com a sua imagem corporal, psicologia $63,3 \%$ e engenharia $94,1 \%$.

Resultados diferentes encontrados no estudo de Claumann et al., (2014) quanto à satisfação com a imagem corporal, observou-se que 79,2\% (n=118) dos acadêmicos apresentaram insatisfação, com proporção mais elevada de acadêmicos insatisfeitos pelo excesso $(53,0 \%)$.

Estudo de Rebolho Martins et al., (2012) ao analisar a insatisfação com a IC por sexo, verificou-se que, entre as mulheres, houve uma maior prevalência de insatisfação com a IC em relação ao excesso de peso $(62,4 \%)$ do que pela magreza $(15,5 \%)$. Entretanto, no sexo masculino, identificou-se uma maior prevalência de insatisfação com a IC em relação à magreza $(43,8 \%)$ do que pelo excesso de peso $(34,1 \%)$.

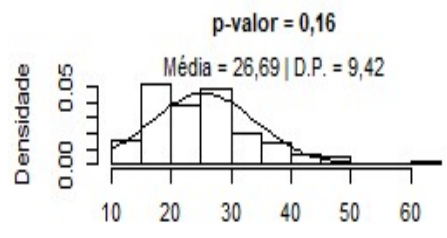

Escore no MBCQ das estud. de Ed. Física

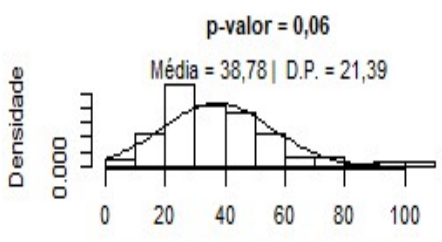

Escore no EAT-26 das estud. de Ed. Física

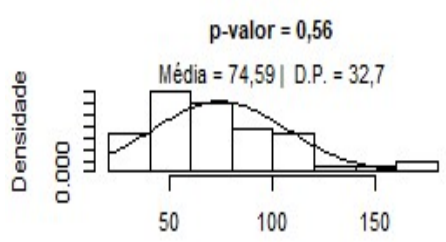

Escore no BSQ das estud. de Ed. Física

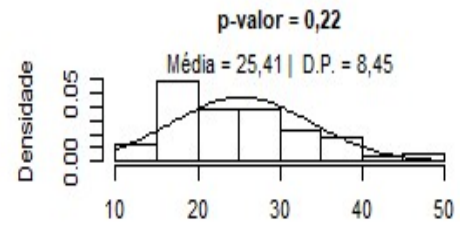

Escore no MBCQ das estud. de Psicologia

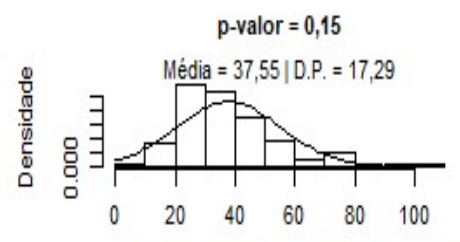

Escore no EAT-26 das estud. de Psicologia

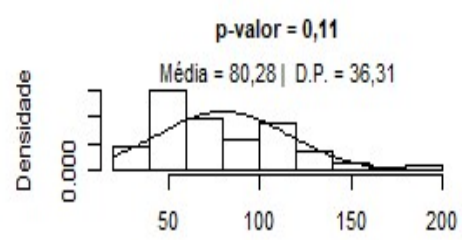

Escore no BSQ das estud. de Psicologia

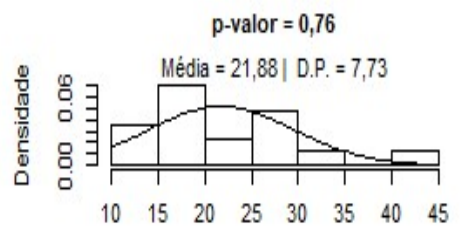

Escore no MBCQ das estud. de Engenharia
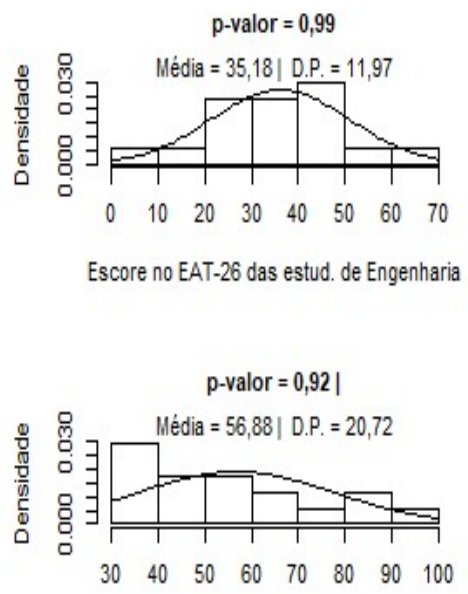

Escore no $M B C Q$ das estud. de Engenharia

Figura 8 - Teste de Kolmogorov- Smirnov para averiguar se os escores obtidos pelas acadêmicas dos cursos de Ed. física, Psicologia e Engenharia para os questionários checagem corporal e comportamento alimentar.

Fonte: Dados da pesquisa, 2019. p-valor $>0,05=$ indicativo que os dados seguem distribuição normal. 
Tabela 4 -Teste t Student entre o curso das acadêmicas e o total de sua pontuação obtida nos questionários MBCQ, EAT-26 e BSQ-34, Teresina, PI, 2019.

\begin{tabular}{|c|c|c|c|c|c|}
\hline Questionários & Curso & $\mathbf{N}$ & Média & D.P.* & p-valor** \\
\hline \multirow{3}{*}{ BCQ } & Ed. Física & 54 & 26,69 & 9,421 & \multirow{3}{*}{0,393} \\
\hline & & & & & \\
\hline & Psicologia & 98 & 25,41 & 8,447 & \\
\hline \multirow{3}{*}{ EAT-26 } & Ed. Física & 54 & 38,78 & 21,39 & \multirow{3}{*}{0,71} \\
\hline & & & & & \\
\hline & Psicologia & 98 & 37,55 & 17,29 & \\
\hline \multirow{3}{*}{ BSQ-34 } & Ed. Física & 54 & 74,59 & 32,699 & \multirow{3}{*}{0,342} \\
\hline & & & & & \\
\hline & Psicologia & 98 & 80,28 & 36,306 & \\
\hline \multirow{3}{*}{ BCQ } & Ed. Física & 54 & 26,69 & 9,421 & \multirow{3}{*}{0,05} \\
\hline & & & & & \\
\hline & Engenharia & 17 & 21,88 & 7,729 & \\
\hline \multirow{3}{*}{ EAT-26 } & Ed. Física & 54 & 38,78 & 21,39 & \multirow{3}{*}{0,511} \\
\hline & & & & & \\
\hline & Engenharia & 17 & 35,18 & 11,98 & \\
\hline \multirow{3}{*}{ BSQ-34 } & Ed. Física & 54 & 74,59 & 32,699 & \multirow{3}{*}{0,04} \\
\hline & & & & & \\
\hline & Engenharia & 17 & 56,88 & 20,721 & \\
\hline \multirow{3}{*}{ BCQ } & Psicologia & 98 & 25,41 & 8,447 & \multirow{3}{*}{0,111} \\
\hline & & & & & \\
\hline & Engenharia & 17 & 21,88 & 7,729 & \\
\hline \multirow{3}{*}{ EAT-26 } & Psicologia & 98 & 37,55 & 17,29 & \multirow{3}{*}{0,588} \\
\hline & & & & & \\
\hline & Engenharia & 17 & 35,18 & 11,98 & \\
\hline \multirow{3}{*}{ BSQ-34 } & Psicologia & 98 & 80,28 & 36,306 & \multirow{3}{*}{0,001} \\
\hline & & & & & \\
\hline & Engenharia & 17 & 56,88 & 20,721 & \\
\hline
\end{tabular}


De acordo com a Tabela 4, tivemos diferença significativa entre as estudantes do curso de Educação Física (pontuação média $\mathrm{BCQ}=26,69$, pontuação média $\mathrm{BSQ} 34=74,59$ ) e as alunas do curso de engenharia (pontuação média $\mathrm{BCQ}=21,88$, pontuação média BSQ-34 = 56,88), referente aos questionários BCQ e BSQ- 34.

Outra diferença significativa houve entre estudantes de psicologia (pontuação média BSQ-34=80,28) e de engenharia (pontuação média BSQ-34 =56,88), ou seja, a um nível de 5\% de significância as estudantes de psicologia se preocupam mais com o corpo do que as estudantes de engenharia.

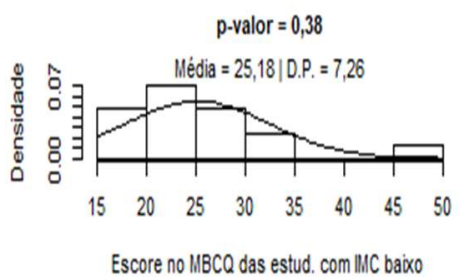

Escore no MBCQ das estud. com IMC baixo

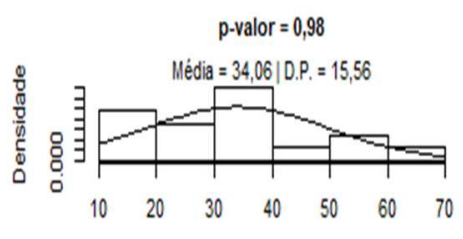

Escore no EAT-26 das estud. com IIIC baixo

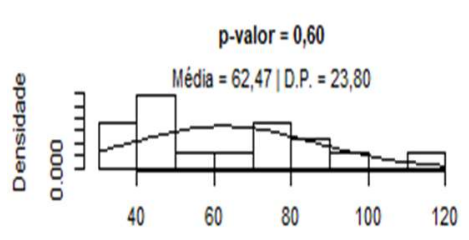

Escore no BSQ das estud. com IIC baixo

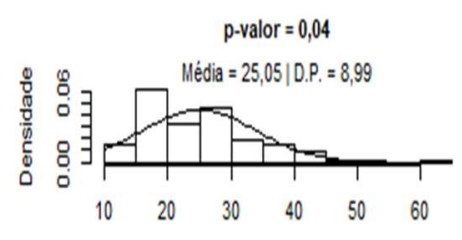

Escore no MBCQ das estud. com IIIC adequado

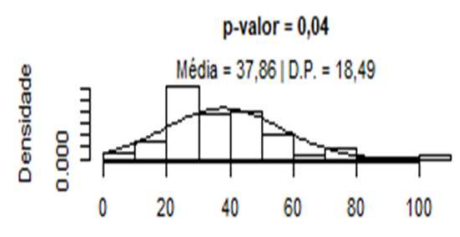

Escore no EAT-26 das estud. com IIIC adequado

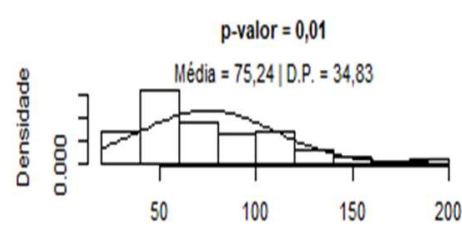

Escore no BSQ das estud. com IIIC adequado

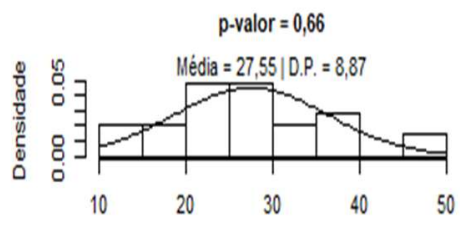

Escore no MBCQ das estud. com IMC acima do peso
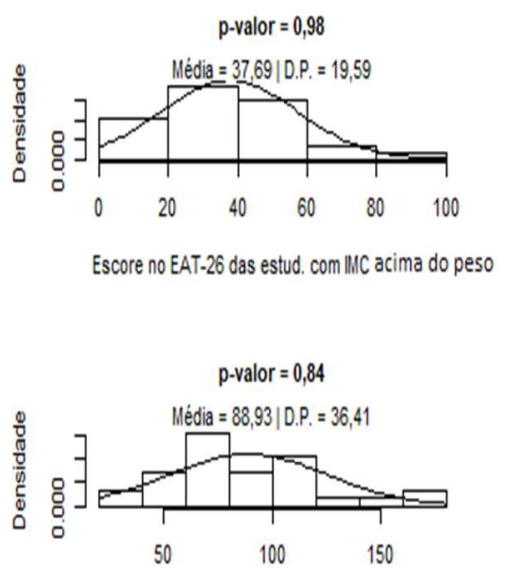

Escore no BSQ das estud. com IIIC acima do peso

Figura 9 - Teste de Kolmogorov- Smirnov para averiguar se os escores obtidos pelas acadêmicas dos cursos de Ed. física, Psicologia e Engenharia, segundo a classificação do IMC, a checagem corporal, comportamento alimentar e satisfação corporal.

Fonte: Dados da pesquisa, 2019.

p-valor $>0,05=$ indicativo que os dados seguem distribuição normal.

Os escores dos questionários para o IMC adequado das acadêmicas não segue a distribuição normal ( $p$-valor $\leq 0,05$ ), ou seja, o teste para esse caso será de U- de Mann Whitney. As demais variáveis o teste usado foi o t-Student, pois os dados seguem a distribuição normal (Figura 10). 
Tabela 5 - Teste t Student entre o IMC das acadêmicas e o total de sua pontuação obtida nos questionários BCQ, EAT-26 e BSQ-34, Teresina, PI, 2019.

\begin{tabular}{|c|c|c|c|c|c|}
\hline Questionários & IMC & $\mathbf{N}$ & Média & D.P.* & p-valor** \\
\hline \multirow{3}{*}{ BCQ } & Baixo peso & 17 & 25,18 & 7,256 & \multirow{3}{*}{0,676} \\
\hline & & & & & \\
\hline & Adequado & 119 & 25,05 & 8,990 & \\
\hline \multirow{3}{*}{ EAT-26 } & Baixo peso & 17 & 34,06 & 15,56 & \multirow{3}{*}{0,431} \\
\hline & & & & & \\
\hline & Adequado & 119 & 37,96 & 18,49 & \\
\hline \multirow{3}{*}{ BSQ-34 } & Baixo peso & 17 & 62,47 & 23,799 & \multirow{3}{*}{0,164} \\
\hline & & & & & \\
\hline & Adequado & 119 & 75,24 & 34,832 & \\
\hline \multirow{3}{*}{ BCQ } & Baixo peso & 17 & 25,18 & 7,256 & \multirow{3}{*}{0,355} \\
\hline & & & & & \\
\hline & Sobrepeso & 29 & 27,55 & 8,870 & \\
\hline \multirow{3}{*}{ EAT-26 } & Baixo peso & 17 & 34,06 & 15,56 & \multirow{3}{*}{0,518} \\
\hline & & & & & \\
\hline & Sobrepeso & 29 & 37,69 & 19,59 & \\
\hline \multirow{3}{*}{ BSQ-34 } & Baixo peso & 17 & 62,47 & 23,799 & \multirow{3}{*}{0,01} \\
\hline & & & & & \\
\hline & Sobrepeso & 29 & 88,93 & 36,412 & \\
\hline \multirow{3}{*}{ BCQ } & Adequado & 119 & 25,05 & 8,990 & \multirow{3}{*}{0,151} \\
\hline & & & & & \\
\hline & Sobrepeso & 29 & 27,55 & 8,870 & \\
\hline \multirow{3}{*}{ EAT-26 } & Adequado & 119 & 37,96 & 18,49 & \multirow{3}{*}{0,956} \\
\hline & & & & & \\
\hline & Sobrepeso & 29 & 37,69 & 19,59 & \\
\hline \multirow{3}{*}{ BSQ -34 } & Adequado & 119 & 75,24 & 34,832 & \multirow{3}{*}{$0,043^{\mathrm{a}}$} \\
\hline & & & & & \\
\hline & Sobrepeso & 29 & 88,93 & 36,412 & \\
\hline
\end{tabular}

Fonte: Dados da pesquisa,2019. 
* Desvio padrão; ** p-valor $\leq 0,05$ é indicativo de diferença entre os grupos; a = utilizou-se o teste não paramétrico U- de Mann Whitney.

$\mathrm{Na}$ Tabela 5, foi relacionado a classificação do IMC com a total da pontuação dos questionários, não utilizamos a classificação obesidade, pois a sua frequência na amostra foi baixa $(n=4)$, o que impossibilita a realização do teste t Student.

Observe que no questionário BSQ-34, houve diferença significativa entre baixo peso (pontuação media $=62,47$ ) e sobrepeso (pontuação média $=88,93$ ), ou seja, ao nível de $5 \%$, as mulheres classificadas como sobrepeso se preocupam mais com a sua forma do corpo, do que as mulheres que estão abaixo do peso e mulheres que estão na classificação adequada do IMC (pontuação média $=75,24$ ).

Conforme a Tabela 5, houve diferença significativa entre os homens (pontuação média $\mathrm{MBCQ}=37,84$, pontuação média EAT-26 = 32,94) e as mulheres (pontuação média BCQ = 25,46, pontuação média EAT-26 = 37,70), referente aos questionários BCQ e EAT-26, que é uma avaliação da checagem corporal e o outro questionário é referente ao comportamento alimentar, ou seja, a um nível de 5\% de significância os homens se preocupam mais com a sua checagem corporal e as mulheres com o comportamento alimentar.
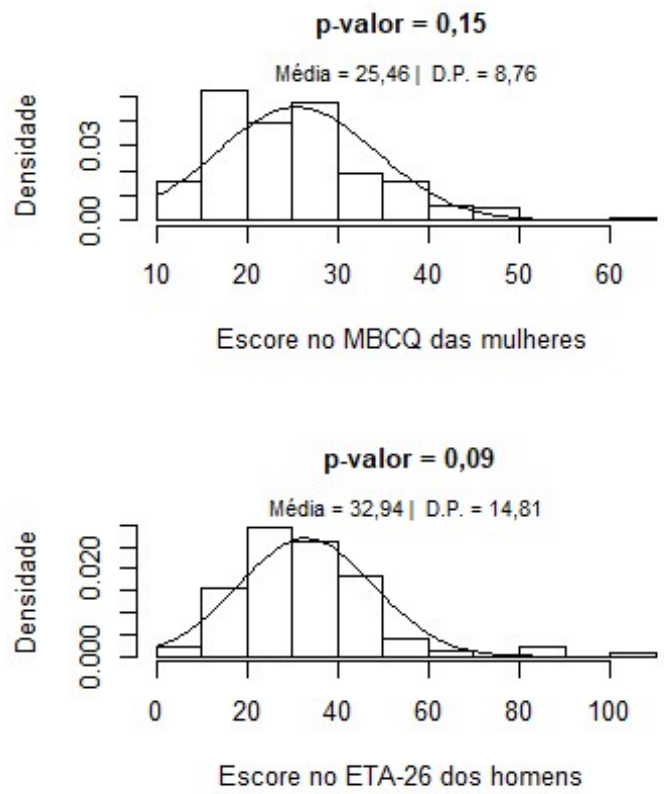
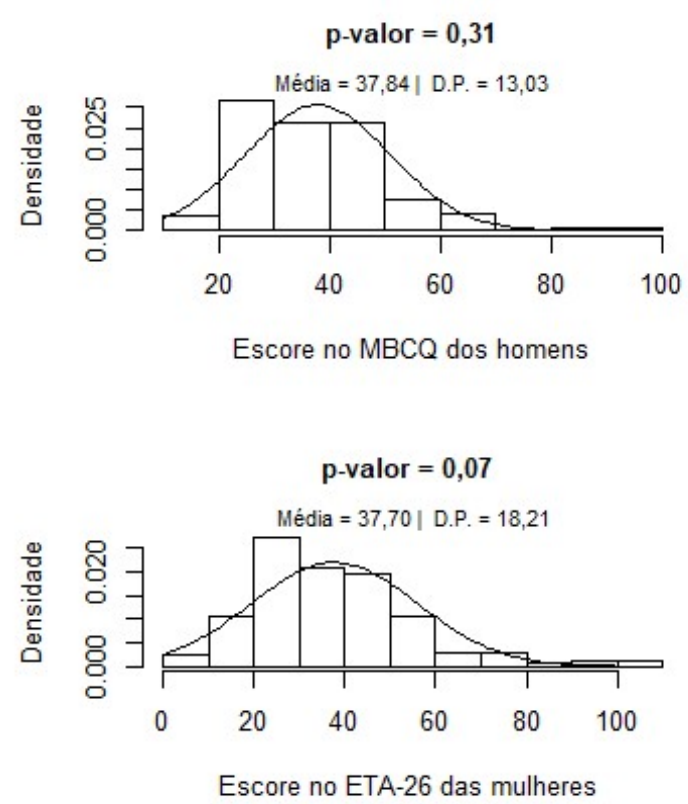

Figura 10 - Teste de Kolmogorov- Smirnov para averiguar se os escores obtidos pelos acadêmicos, para a checagem corporal e comportamento alimentar.

Fonte: Dados da pesquisa, 2019. p-valor $>0,05=$ indicativo que os dados seguem distribuição normal. 
Tabela 6 - Teste t Student entre o sexo e o total de sua pontuação obtida nos questionários MBCQ e EAT-26, Teresina, PI, 2019.

\begin{tabular}{lllccc}
\hline Questionários & Sexo & N & Média & D.P.* & p-valor** \\
\hline MBCQ & Masculino & 146 & 37,84 & 13,028 & \\
BCQ & Feminino & 169 & 25,46 & 8,756 & $<\mathbf{0 , 0 0 1}$ \\
\hline \multirow{2}{*}{ EAT-26 } & Masculino & 146 & 32,94 & 15,541 & \\
& Feminino & 169 & 37,70 & 18,206 & $\mathbf{0 , 0 0 7}$
\end{tabular}

Fonte: Dados da pesquisa, 2019. * Desvio padrão; ** p-valor $\leq 0,05$ é indicativo de diferença entre os grupos.

Observou-se que não houve nenhuma diferença significativa $(p \leq 0,05)$, então com um nível de 5\% não obteve relação entre a checagem corporal e comportamento alimentar.

\section{Conclusão}

O presente estudo permite concluir que o sexo feminino apresentou maior prevalência de adequação em relação ao índice de massa corporal quando comparadas ao sexo masculino, já em relação à percepção da imagem corporal, observou-se que ambos os sexos apresentam baixa frequência de comportamento de checagem corporal. Quanto ao comportamento alimentar, as mulheres obtiveram maiores pontuações que os homens, o que sugere uma maior suscetibilidade para o desenvolvimento de TAs. Em relação a satisfação corporal o curso de engenharia obteve-se maior nível de satisfação corporal.

Novos estudos avaliando percepção da imagem corporal e comportamento alimentar são importantes. Tais pesquisas poderiam não apenas contribuir para melhor formação desses futuros profissionais, mas também incentivariam ações preventivas que poderiam auxiliar na conscientização dos estudantes a terem uma melhor aceitação do seu próprio corpo. 


\section{Referências}

AMARAL, A.C.S.et al. Imagem Corporal de adolescentes: descrição e intervenção preventiva em âmbito escolar. Tese (Doutorado em Psicologia) 2015. 260 f. Instituto de Ciências Humanas Universidade Federal de Juiz De Fora MG , 2015.

ALMEIDA, A. B. P. et al. Avaliação do comportamento alimentar de estudantes universitários. Nutrire. São Paulo, v. 38, p. 411, 2013.

ALVES, D.; PINTO, M.; ALVES, S.; MOTA, A.; LEIRÓS, V. Cultura e imagem corporal. Motricidade, Santa Maria da Feira, v. 5, n. 1, p. 1-20, 2009.

ALVARENGA, M.S.; SCAGLIUSI, F. B.; PHILIPPI, S. T. Comportamento de risco para transtorno alimentar em universitárias brasileiras. Rev. Psiquiatr. Clín.v.38, n.1,p.3-7, 2011.

ASSIS, C. L. et al. Autoavaliação de peso corporal e classificação do índice de massa corporal de estudantes do Ensino Superior de Cacoal-RO. Mudanças-Psicologia da Saúde, v. 21, n. 2, p. 30-39, 2013.

CLAUMANN, G.S. et al. Satisfação com a imagem corporal em acadêmicos ingressantes em cursos de educação física. Revista de Educação Física, v. 25, n. 4, p. 575-583, 2014.

FERREIRA, M.E.C. et al. Checagem corporal, atitude alimentar inadequada e insatisfação com a imagem corporal de jovens universitários. Jornal Brasileiro de Psiquiatria, v.62, n.2, p.108-114, 2013.

GASPARETTO, R.M. et al. Perfil antropométrico dos universitários dos cursos de nutrição, enfermagem, fisioterapia e educação física do Centro Universitário La Salle,

Canoas/RS. Revista da Associação Brasileira de Nutrição-RASBRAN, v. 4, n. 1, p. 29-33, 2012.

LAUS, M.F.; MOREIRA, R.C. M.; COSTA, T.M. B. Diferenças na percepção da imagem corporal, no comportamento alimentar e no estado nutricional de universitárias das áreas de saúde e humanas. Rev Psiquiatr Rio Gd Sul, v. 31, n. 3, p. 192-6, 2009.

MOURA DE ARAÚJO, M.F. et al. Avaliação da qualidade do sono de estudantes universitários de Fortaleza-CE. Texto \& contexto enfermagem, v. 22, n. 2, 2013.

REBOLHO MARTINS, C. al. Insatisfação com a imagem corporal e fatores associados em universitários. Estudos de Psicologia, v. 17, n. 2, 2012.

SILVA, L. S. Insatisfação e percepção da imagem corporal e sua associação com o índice de massa corporal em estudantes de ensino médio de escolas públicas de Porto

Alegre/RS. (TCC) Universidade Federal do Rio Grande do Sul. Faculdade de Medicina. Curso de Nutrição.RS, 2012.

SILVA, K.M.a. Hábitos alimentares em acadêmicos do curso de nutrição. 2013.

Sample size calculator by Raosoft. Inc. disponível gratuitamente no endereço eletrônico www.Raosoft.com. 


\title{
PERCEPTION OF BODY IMAGE AND EATING BEHAVIOR: A STUDY WITH UNIVERSITY STUDENTS
}

\begin{abstract}
The concept of corporal image includes how own body, taking into account their constituent parts, as well as the feelings related the those characteristics. The entrance in academica life provokes alterations in the feeding behavior, and the exposition to the new social environment they can bring as consequence an increase in dissatisfaction. Objective: To investigate the satisfaction with the body image and eating behavior of academics at a university center. Methods: For data collection, the following instruments were used. The evaluation was performed with anthropometric measurements, as size and weight measurements. In order to diagnose the nutritional status, the body mass index was used. In order to evaluate the study variables; we used the Body Checking questionnaires Questionnaire. Male Body Checking Questionnaire, Body Shape Questionnaire and EAT-26. Results: According to the Body Mass Index, the female gender had a higher rate of adequacy $(70.4 \%)$, in relation to the masculine $(58,9 \%)$. The EAT-26 questionnaire showed that $85 \%$ of male engineering students presented risk for Feeding Disorders, in relation to the female psychology (89.8\%). Conclusion: In terms of feeding behavior, the women scored higher than men, which suggests a higher susceptibility to Feeding Disorders development in men.
\end{abstract}

Keywords: Body Image; Nutritional Assessment; Food Behavior 
ANEXO A - PARECER CONSUBSTANCIADO DO CEP

ASSOCIAÇÃO TERESINENSE
DE ENSINO - FACULDADES
SANTO AGOSTINHOATE

\section{PARECER CONSUBSTANCIADO DO CEP}

\section{DADOS DO PROJETO DE PESQUISA}

Titulo da Posquisa CHECAGEM CORPDRAL E COMPORTAMENTO ALIMENTAR, ESTUDO COM ACADEMICOS DE ZENTRO UNIVERSITRRIO

Pesquisador: LULAA MARLY FREITAS DE CARVALHO

Area Tombtica:

Versac: 2

CAAE: 91003018.00000 .5602

Instituiço Proponente: SSSOCLAÇÃO TERESINENSE DE ENSNO SC LTDA

Patrocinador Prindpiat: Financiamemta Poprio

\section{DADOS DO PARECER}

Número do Parocer: 2.845.870

\section{Apresontaça do Frojotoc}

O estudo da pesculsadora responsdvi Ms. LUaZA MARLY FREITAS DE CAFVALHO, O demais pesquisadores WATEUS EARROS IE CARVALHO E FAULA MERIA DA \$LVA. NE CAAE

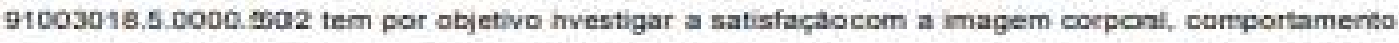
alimentar de acadenticos de um centro unversitario. Para a calea de dados serâ ueievados os seguintes instrumentos: a avalaucalo nutricianal sera ealirada atraves de attropornetrica, com as neidax de peso $\mathrm{e}$ estahura, para diogidestico do eatodo nutracnal sera utilizodo olMK; para avaliar os omportamentos de checagem do corpe no sexo feminino, serb utilzado a Bochy Checking Ouestiannaire (ECO); para avaliar a checagem corporil dos individuos dasexo masculino seri realizada peio Mak Body Checking Ouestionnaire(MBCO); para avaliar o conpartamento alimenta sera avaliado pela aulicapa da vers.Sa tradumita do teste o atitudes alimentares EAT 26k e para avaliz a insatisfaça e as peccupopoes coen a forma do corpo, sed utilcado o Eody Shape Questionnaire (ESQ). Trata-se de estudo de campo com ahordagem transversal quantitabvo e desintivo a ser realizada ro Centro Universithriolacalizado na zona urbana e regisa sulda cidade de Teresine. Piaul. O eshido serarealizado cam acadenicas de um Centro Universitario, com jopulaça total de 1761 academioos e amasta total de 316 individuos distribuidos noe cursas de Engenhaia Covil, Psicologia e Educopso Fisica. A amotra fai determinada peb programa sampie size calculator by Racactt. Inc. disponiveigratuitamente no endereço eletrönico www. Ifasosaft com. O nivel de significancia de $35 \%$ e margem de erre de $5 \%$.

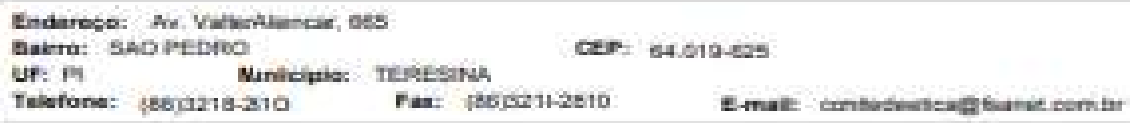

